Classification

Physics Abstracts

73.90

\title{
Dopage par As de couches épitaxiées de Ge sur substrats Ge et GaAs
}

\author{
D. Etienne, N. Achargui et G. Bougnot \\ Centre d'Electronique de Montpellier (UA.391), Université des Sciences et Techniques du Languedoc, \\ Place E. Bataillon, 34060 Montpellier Cedex, France
}

(Reçu le 27 mars 1985, révisé le 22 octobre 1985, accepté le 28 janvier 1986)

\begin{abstract}
Résumé. - Des dépôts de Ge dopés As sont obtenus par transport en phase vapeur par la réaction de disproportionnation : $2 \mathrm{GeI}_{2}=\mathrm{Ge}+\mathrm{GeI}_{4}$. Ils sont de type $\mathrm{n}$ et leurs caractéristiques électriques $: \rho, \mu_{\mathrm{H}}, \mathrm{n}$ sont étudiés en fonction de la température de substrat et du débit de $\mathrm{AsH}_{3}$. L'incorporation de As dans la couche est étudiée thermodynamiquement.
\end{abstract}

Abstract. - As doped Ge layers were obtained by chemical vapour transport using a disproportiounation reaction $2 \mathrm{GeI}_{2}=\mathrm{Ge}+\mathrm{GeI}_{4}$. They were $\mathrm{n}$ type and their electric parameters $\rho, \mu_{\mathrm{H}}, \mathrm{n}$ were studied as a function of substrate temperature and flow rate of $\mathrm{AsH}_{3}$. The incorporation of As into the epilayer is studied thermodynamically.

\section{Introduction.}

Le germanium dont l'étude fondamentale a été abandonnée depuis quelques années est susceptible de retrouver un certain intérêt dans un axe photovoltaïque et en particulier dans un système de cellules photovoltaïques multicolores dont on peut espérer un rendement de conversion élevé.

Dans ce but, deux méthodes de conversion photovoltaïque multicolore peuvent être utilisées :

- le système à photopiles spectralement distribuées dont le tandem Ge-GaAs peut être une première étape,

- l'empilement de cellules en série électrique et optique dans lequel une photopile «arc-en-ciel » monolithique du type Ge-GaAs-GaAlAs peut être retenue.

De ce fait, l'étude de la faisabilité d'une jonction p-n au Ge pouvait être envisagée et elle devait répondre à deux critères :

- contrôle du taux de dopage intentionnel introduit dans les couches,

- épitaxie effectuée à température suffisamment basse afin d'éviter l'interdiffusion des éléments et la dégradation de la jonction p-n dans GaAs.

La technique utilisée et qui répond le mieux à ces critères est le transport en phase vapeur en tube ouvert utilisant l'iode en tant qu'agent réactif. Cette technique permet des températures de croissance de l'ordre de $350^{\circ} \mathrm{C}$ [1 à 8].

Dans le cadre de cette étude, nous nous sommes intéressés au contrôle du dopage $\mathrm{n}$ par introduction de $\mathrm{AsH}_{3}$ dilué dans $\mathrm{H}_{2}$, dans la phase vapeur et en particulier quels pouvaient être les niveaux de dopage atteints. Les couches de Ge dopées ont été élaborées sur substrats Ge et GaAs et nous avons étudié l'évolution des propriétés électriques : mobilité, résistivité et nombre de porteurs en fonction des paramètres propres à la technique de croissance, c'est-à-dire température de dépôt et débit de $\mathrm{AsH}_{3}$ introduit dans la phase vapeur.

\section{Dispositif expérimental.}

Un flux de $\mathrm{H}_{2}+\mathrm{He}$ entraîne des vapeurs̄ de $\mathrm{I}_{2}$ sur une source de Ge maintenue à température $T_{1}$. Les vapeurs de $I_{2}$ sont obtenues à partir d'une source d'iode maintenue à température constante. La source est constituée par du Ge intrinsèque polycristallin, de résistivité $40-50 \Omega . \mathrm{cm}$.

Les substrats sont du $\mathrm{Ge}(\mathrm{p})$ d'orientation (111), $\rho=40 \Omega$.cm et du GaAs semi-isolant d'orientation (100). Ces substrats sont les seuls que nous ayons utilisés car ils ont des résistivités élevées et ne perturbent pas les mesures électriques effectuées sur la couche. Le dopage $n$ est assuré par de l'arsine dilué à $100 \mathrm{ppm}$ dans l'hydrogène.

\section{Résultats expérimentaux.}

3.1 CARACTÉRISATION DES DÉPÔTS.

3.1.1 Morphologie des couches. - La photographie 1 représente un exemple de dépôt $\mathrm{Ge}(\mathrm{n}) / \mathrm{GaAs}$. Dans tous les cas, nous obtenons une structure bien définie, 
l'aspect étant sensiblement identique à celui des couches naturelles obtenues dans des conditions analogues.

La photographie 2 représente un exemple $\mathrm{Ge}(\mathrm{n}) / \mathrm{Ge}$. Nous obtenons également dans ce cas, un aspect identique à celui des couches non dopées.

3.1.2 Etude en diffraction électronique. - Cette méthode de caractérisation a été utilisée systématiquement sur tous les échantillons $\mathrm{Ge}(\mathrm{n})$ obtenus sur substrat $\mathrm{Ge}$ et GaAs. Appliquée, en fait, qualitativement elle permet d'estimer l'homogénéité des dépôts,

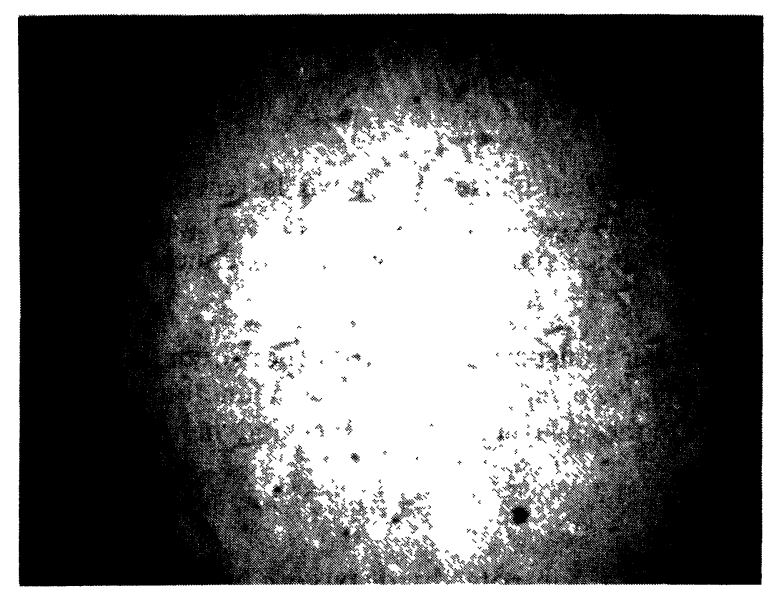

Photo. 1. - Etat de surface d'un dépôt $\mathrm{Ge}(\mathrm{n}) / \mathrm{Ge}$ pour : $T_{\text {substrat }}=340^{\circ} \mathrm{C} ; T_{\text {source }}=570^{\circ} \mathrm{C} ; T_{\mathrm{I}_{2}}=75^{\circ} \mathrm{C} ; d_{\mathrm{H}_{2}}=$ $25 \mathrm{cc} / \mathrm{min} ; d_{\mathrm{He}}=225 \mathrm{cc} / \mathrm{min} ; d_{\mathrm{AsH}_{3}}=2 \mathrm{cc} / \mathrm{min}$.

[Surface morphology of $\mathrm{Ge}(\mathrm{n}) / \mathrm{Ge}$ for : $T_{\text {substrate }}=340{ }^{\circ} \mathrm{C}$; $T_{\text {source }}=570^{\circ} \mathrm{C} ; T_{\mathrm{I}_{2}}=75^{\circ} \mathrm{C} ; d_{\mathrm{H}_{2}}=25 \mathrm{cc} / \mathrm{min} ; d_{\mathrm{He}}=$ $225 \mathrm{cc} / \mathrm{min} ; d_{\mathrm{AsH}_{3}}=2 \mathrm{cc} / \mathrm{min}$.]

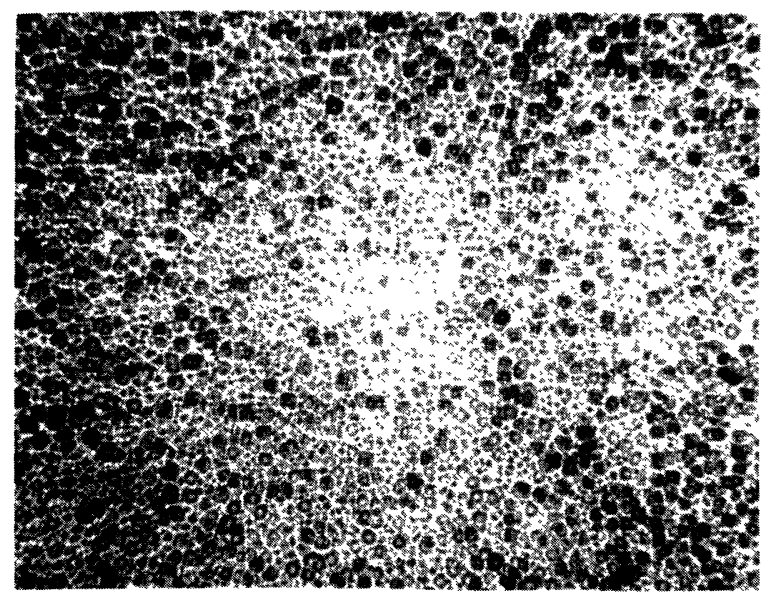

Photo. 2. - Etat de surface d'un dépôt $\mathrm{Ge}(\mathrm{n}) / \mathrm{GaAs}$ pour : $T_{\text {substrat }}=345^{\circ} \mathrm{C} ; T_{\text {source }}=570{ }^{\circ} \mathrm{C} ; T_{\mathrm{I}_{2}}=75^{\circ} \mathrm{C} ; d_{\mathrm{H}_{2}}=$ $25 \mathrm{cc} / \mathrm{min} ; d_{\mathrm{He}}=225 \mathrm{cc} / \mathrm{min} ; d_{\mathrm{AsH}_{3}}=2 \mathrm{cc} / \mathrm{min}$.

[Surface morphology of $\mathrm{Ge}(\mathrm{n}) / \mathrm{GaAs}$ for : $T_{\text {substrate }}=$ $345^{\circ} \mathrm{C} ; T_{\text {source }}=570{ }^{\circ} \mathrm{C} ; T_{\mathrm{I}_{2}}=75^{\circ} \mathrm{C} ; d_{\mathrm{H}_{2}}=25 \mathrm{cc} / \mathrm{min}$; $d_{\mathrm{He}}=225 \mathrm{cc} / \mathrm{min} ; d_{\mathrm{AsH}_{3}}=2 \mathrm{cc} / \mathrm{min}$.] d'autant qu'elle ne concerne que leur surface et non le volume. Toutes les diffractions réalisées sur les dépôts $\mathrm{Ge}(\mathrm{n}) / \mathrm{Ge}$ et $\mathrm{Ge}(\mathrm{n}) / \mathrm{GaAs}$ dans les conditions de croissance normales et en présence de $\mathrm{AsH}_{3}+\mathrm{H}_{2}$ dans la zone de dépôt sont reproductibles et montrent que les couches ne présentent pas de maclage et sont monocristallines.

\subsection{Caractérisation ÉleCtriQue.}

3.2.1 Etude sur substrats GaAs. - Nous avons représenté sur la figure 1 la variation de la résistivité, de la mobilité et du nombre de porteurs en fonction de la température de dépôt.

La première constatation concerne le fort taux de dopage observé avec les conditions que nous utilisons : en effet la concentration en impuretés varie de $7 \times 10^{18}$ à $2,5 \times 10^{21} \mathrm{~cm}^{-3}$ pour une gamme de température assez restreinte.

Le nombre de porteurs présente un minimum de l'ordre de $7 \times 10^{18} \mathrm{~cm}^{-3}$ à une température de dépôt de $330^{\circ} \mathrm{C}$, c'est-à-dire à une température inférieure à la température optimale de dépôt en l'absence de dopage [9]. Ce minimum est probablement dû à la compétition entre les réactions de formation de $\mathrm{Ge}$ et As à partir des iodures. Ce décalage en température pouvant provenir du fait que le dopage en arsenic se superpose au dopage résiduel par le substrat, qui croît lorsque la température de dépôt augmente [10].

Aux températures élevées, la teneur en As devient très grande, $n \simeq 10^{21} \mathrm{~cm}^{-3}$, il semble alors se former

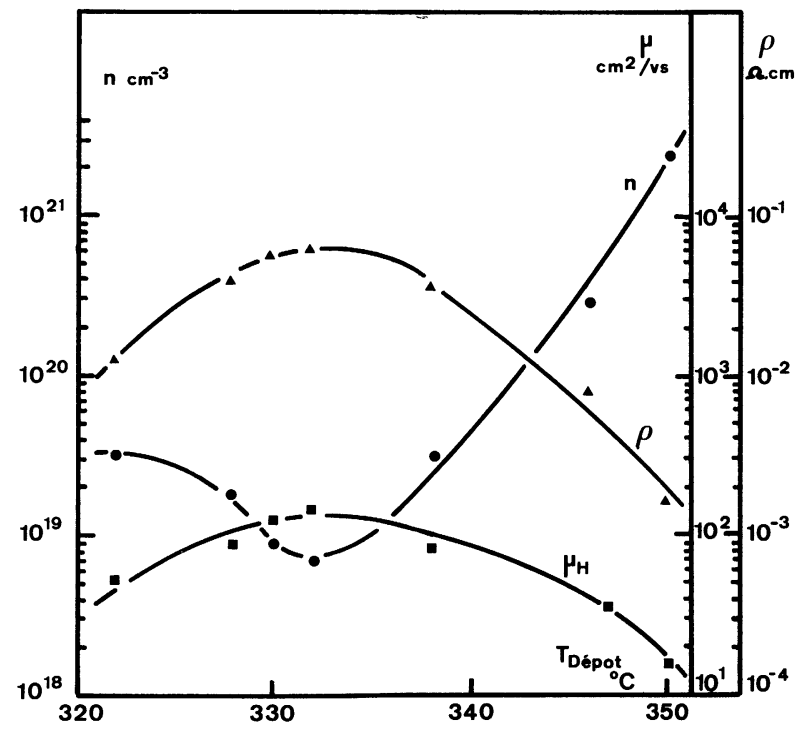

Fig. 1. - $\mathrm{Ge}(\mathrm{n}) / \mathrm{GaAs}$. - Variation de la résistivité, de la mobilité et du nombre de porteurs en fonction de la température de dépôt pour $T_{\text {source }}=590^{\circ} \mathrm{C} ; F=0,1\left(d_{\mathrm{H}_{2}}=\right.$ $\left.25 \mathrm{cc} / \mathrm{min} ; d_{\mathrm{He}}=225 \mathrm{cc} / \mathrm{min}\right) d_{\mathrm{AsH}_{3}}=2 \mathrm{cc} / \mathrm{min} ; T_{\mathrm{I}_{2}}=75^{\circ} \mathrm{C}$.

[Ge(n)/GaAs. Resistivity, hole mobilities and hole concentration as a function of substrate temperature for : $T_{\text {source }}=590^{\circ} \mathrm{C} ; F=0.1\left(d_{\mathrm{H}_{2}}=25 \mathrm{cc} / \mathrm{min}, d_{\mathrm{He}}=225 \mathrm{cc} /\right.$ $\mathrm{min}) ; d_{\mathrm{AsH}_{3}}=2 \mathrm{cc} / \mathrm{min} ; T_{\mathrm{I}_{2}}=75^{\circ} \mathrm{C}$.] 
un véritable alliage Ge-As. En fonction du débit de $\mathrm{AsH}_{3}$ admis dans la phase vapeur, la variation du nombre de porteurs est reporté sur la figure 2 .

On remarque que la concentration en donneurs augmente peu lorsqu'on augmente le débit de $\mathrm{AsH}_{3}$, cette saturation implique que même à faible débit on atteint un taux maximum d'incorporation d'atomes d'arsenic.

3.2.2 Etude sur substrats Ge. - L'allure générale de l'évolution du nombre de porteurs en fonction de la température de dépôt (Fig. 3) est sensiblement la même que dans le cas du substrat GaAs, mais son minimum s'est déplacé vers des températures supérieures $\left(\sim 350^{\circ} \mathrm{C}\right)$, ceci provient du fait qu'ici le dopage ne s'ajoute pas au dopage résiduel par l'arsenic provenant du substrat, du fait que l'on dépose sur un substrat de Ge.

On note également les faibles valeurs de la mobilité, ce qui entraîne des couches plus résistives que celles obtenues sur substrat GaAs. En fonction du débit de $\mathrm{AsH}_{3}$ arrivant dans la zone de dépôt la variation du nombre de porteurs est reporté sur la figure 4 .

\section{Interprétation des résultats.}

Nous nous trouvons en présence au niveau de la zone de dépôt, des espèces chimiques en phase vapeur provenant de la zone source soit : $\mathrm{H}_{2}, \mathrm{I}, \mathrm{He}, \mathrm{I}_{2}, \mathrm{HI}$, $\mathrm{GeI}_{2}$ et des espèces chimiques introduites pour le dopage soit $\mathrm{AsH}_{3}$ dilué dans $\mathrm{H}_{2}$.

$\mathrm{Ce}$ que nous pouvons schématiser de la façon suivante :

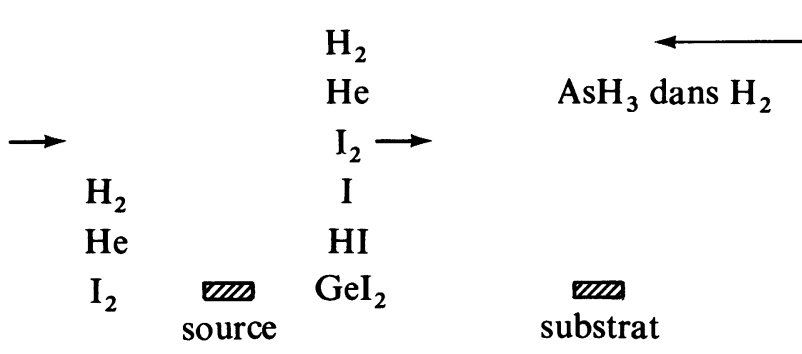

Compte tenu de ces espèces chimiques en présence, nous pouvons écrire les réactions chimiques susceptibles de se former :

- décomposition thermique de $\mathrm{AsH}_{3}$

$$
\mathrm{AsH}_{3}(\mathrm{~g}) \leftrightarrows \mathrm{As}(\mathrm{s})+3 / 2 \mathrm{H}_{2}(\mathrm{~g})
$$

- réactions chimiques mettant en jeu $\mathrm{AsH}_{3}$ et $\mathrm{I}_{2}$ ou les composés iodés :

$$
\begin{aligned}
\mathrm{AsH}_{3}(\mathrm{~g})+3 / 2 \mathrm{I}_{2}(\mathrm{~g}) & \leftrightarrows \mathrm{AsI}_{3}(\mathrm{~g})+3 / 2 \mathrm{H}_{2}(\mathrm{~g}) \\
\mathrm{AsH}_{3}(\mathrm{~g})+3 \mathrm{HI}(\mathrm{g}) & \hookrightarrow \mathrm{AsI}_{3}(\mathrm{~g})+3 \mathrm{H}_{2}(\mathrm{~g})
\end{aligned}
$$

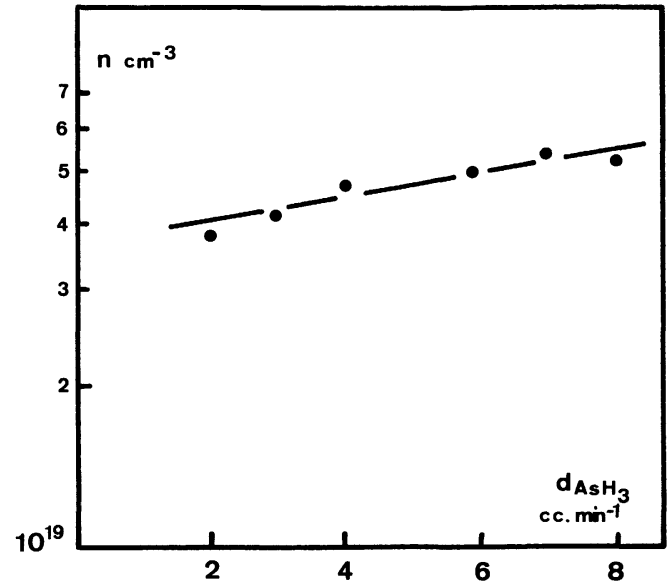

Fig. 2. - Ge(n)/GaAs. - Variation du nombre de porteurs en fonction du débit de $\mathrm{AsH}_{3}$ avec : $T_{\text {source }}=590^{\circ} \mathrm{C}$; $T_{\text {dépot }}=338^{\circ} \mathrm{C} ; F=0,1\left(d_{\mathrm{H}_{2}}=25 \mathrm{cc} / \mathrm{min}, d_{\mathrm{He}}=225 \mathrm{cc} /\right.$ $\min ) ; T_{\mathrm{I}_{2}}=75^{\circ} \mathrm{C}$.

[Ge(n)/GaAs. Hole concentration as a function of $\mathrm{AsH}_{3}$ flow for : $T_{\text {source }}=590^{\circ} \mathrm{C} ; T_{\text {substrate }}=338^{\circ} \mathrm{C} ; F=0.1$ $\left(d_{\mathrm{H}_{2}}=25 \mathrm{cc} / \mathrm{min}, d_{\mathrm{He}}=225 \mathrm{cc} / \mathrm{min}\right) ; T_{\mathrm{I}_{2}}=75^{\circ} \mathrm{C}$. $]$

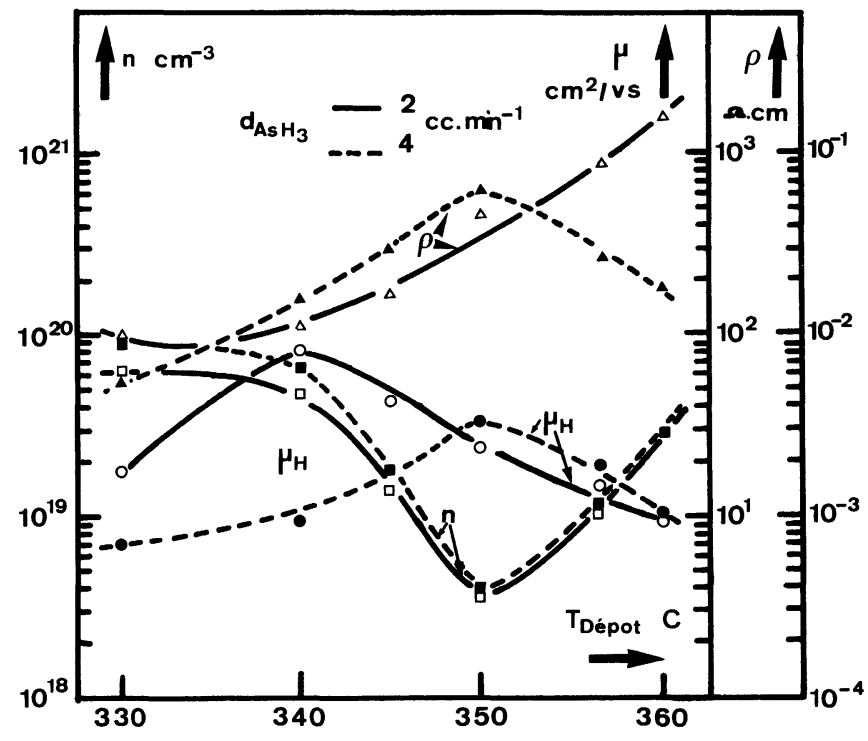

Fig. 3. - Ge(n)/Ge. - Variation du nombre de porteurs en fonction de la température de dépôt pour : $T_{\text {source }}=$ $590^{\circ} \mathrm{C} ; F=0,1 \quad\left(d_{\mathrm{H}_{2}}=25 \mathrm{cc} / \mathrm{min}, d_{\mathrm{He}}=225 \mathrm{cc} / \mathrm{min}\right)$; $T_{\mathrm{I}_{2}}=75^{\circ} \mathrm{C} ; d_{\mathrm{AsH}_{3}}=2$ et $4 \mathrm{cc} / \mathrm{min}$.

$[\mathrm{Ge}(\mathrm{n}) / \mathrm{Ge}$. Hole concentration as a function of substrate temperature for : $T_{\text {source }}=590^{\circ} \mathrm{C} ; F=0.1\left(d_{\mathrm{H}_{2}}=25 \mathrm{cc} /\right.$ $\left.\min , d_{\mathrm{He}}=225 \mathrm{cc} / \mathrm{min}\right) ; T_{\mathrm{I}_{2}}=75^{\circ} \mathrm{C} ; d_{\mathrm{AsH}_{3}}=2$ et $4 \mathrm{cc} /$ min.]

- réactivité de $\mathrm{AsI}_{3}$ en présence de $\mathrm{GeI}_{2}$

$$
\mathrm{AsI}_{3}(\mathrm{~g})+3 / 2 \mathrm{GeI}_{2}(\mathrm{~g}) \leftrightarrows \mathrm{As}(\mathrm{s})+3 / 2 \mathrm{GeI}_{4}(\mathrm{~g})
$$

Nous allons déterminer la fraction $\beta$ de $\mathrm{AsI}_{3}$ qui est réduit par $\mathrm{GeI}_{2}$ suivant la réaction (4). Pour 


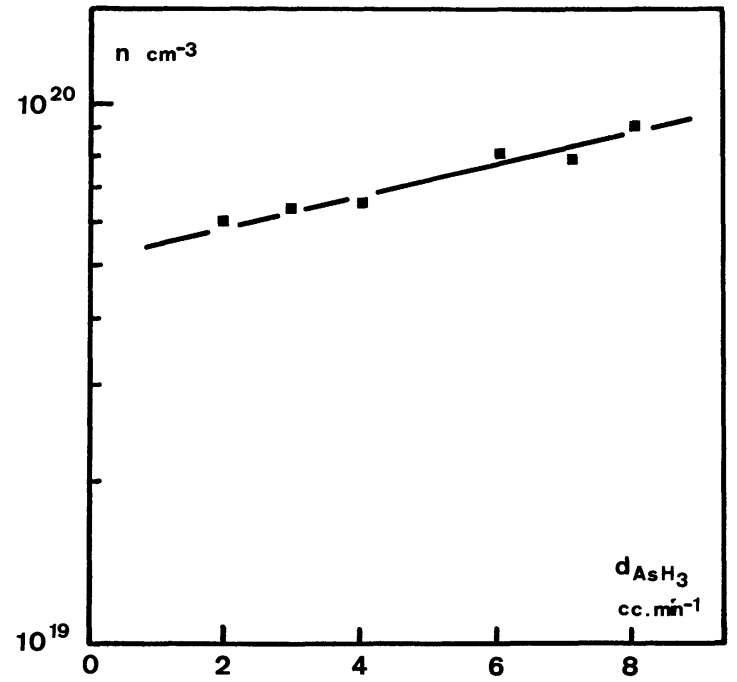

Fig. 4. - $\mathrm{Ge}(\mathrm{n}) / \mathrm{Ge}$ - - Variation du nombre de porteurs en fonction du débit de $\mathrm{AsH}_{3}$ pour : $T_{\text {source }}=590^{\circ} \mathrm{C}$; $T_{\text {dépot }}=330^{\circ} \mathrm{C} ; F=0,1\left(d_{\mathrm{H}_{2}}=25 \mathrm{cc} / \mathrm{min}, d_{\mathrm{He}}=225 \mathrm{cc} /\right.$ $\min ) T_{I_{2}}=75^{\circ} \mathrm{C}$.

[Ge(n)/Ge. Hole concentration as a function of $\mathrm{AsH}_{3}$ flow for $T_{\text {source }}=590^{\circ} \mathrm{C} ; T_{\text {substrat }}=330{ }^{\circ} \mathrm{C} ; F=0.1\left(d_{\mathrm{H}_{2}}=\right.$ $\left.25 \mathrm{cc} / \mathrm{min} ; d_{\mathrm{He}}=225 \mathrm{cc} / \mathrm{min}\right) ; T_{\mathrm{I}_{2}}=75^{\circ} \mathrm{C}$.]

cela à partir de la valeur de $K$ de la réaction (4) faisant intervenir les pressions partielles et en introduisant les pressions partielles initiales des espèces $\mathrm{AsI}_{3}(\mathrm{~g})$ et $\mathrm{GeI}_{2}(\mathrm{~g})$ nous pouvons écrire :

$$
K(4)=\frac{\beta^{3 / 2} P_{\mathrm{Asl}_{3}}^{* 1 / 2}}{(1-\beta)\left[2 / 3 P_{\mathrm{GeI}_{2}}-\beta P_{\mathrm{AsI}_{3}}^{*}\right]^{3 / 2}} .
$$

La variation de $\beta$ en fonction de $\log K$ est représentée sur la figure 5 , les courbes étant paramétrées suivant différentes valeurs des pressions initiales de $\mathrm{AsI}_{3}$ et $\mathrm{GeI}_{2}$.

A partir de la relation liant la constante d'équilibre de la réaction $K$ à la variation d'énergie libre standard $\Delta G_{\mathrm{T}}^{0}$ :

$$
R . T \log K=-\Delta G_{\mathrm{T}}^{0}
$$

on peut représenter la variation de $\log K$ en fonction de $\Delta G_{\mathrm{T}}^{0}$ pour différentes températures.

En présence de cette variation, on peut donc en déduire la variation de $\beta$ en fonction de $T$. Nous obtenons que $\beta$ est égal à 1 à basse température et devient légèrement inférieur à 1 à haute température. C'est-à-dire que $\mathrm{AsI}_{3}$ est fortement réduit par $\mathrm{GeI}_{2}$ suivant la réaction (4) avec libération de As.

Nous sommes donc en présence au niveau de la zone de dépôt d'une importante quantité de As provenant d'une part de la décomposition thermique de $\mathrm{AsH}_{3}$, d'autre part de la formation de $\mathrm{AsI}_{3}$ et de sa réactivité en présence de $\mathrm{GeI}_{2}$. Notons également que l'on doit envisager la réactivité du substrat $\mathrm{GaAs}$ avec les espèces gazeuses présentes dans le système

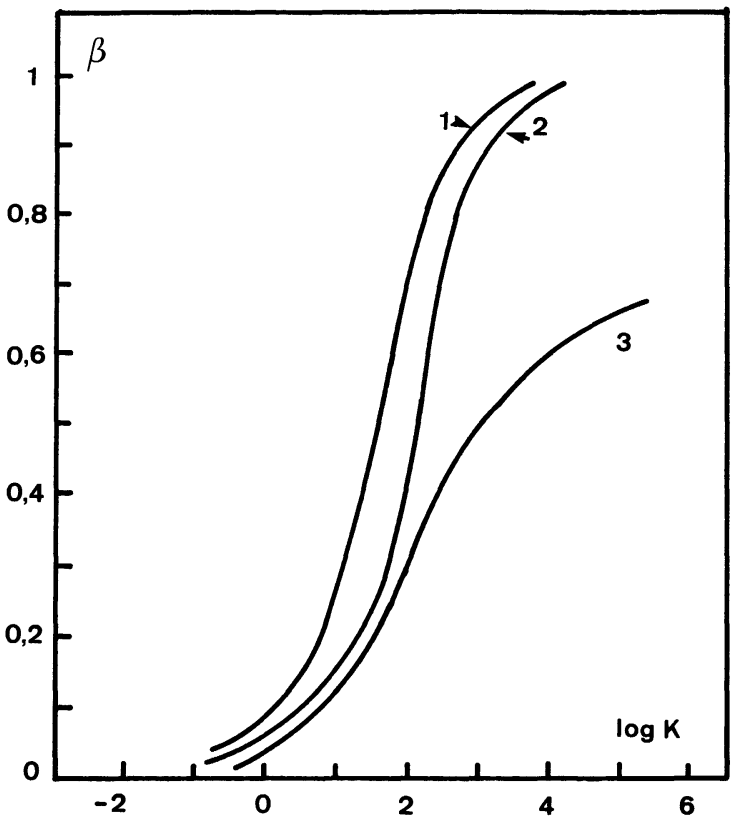

Fig. 5. - Variation de $\beta$ en fonction de $\log$ K. 1. $P_{\mathrm{AsI}_{3}}^{*}=$ $1,3 \times 10^{-3} \mathrm{~atm} ; \quad P_{\mathrm{Gel}_{2}}^{*}=1,3 \times 10^{-2} \mathrm{~atm} ; 2 . P_{\mathrm{AsI}_{3}}^{*}=$ $1,3 \times 10^{-3}$ atm; $P_{\mathrm{GeI}_{2}}^{*}=6,6 \times 10^{-3} \mathrm{~atm} ; 3 . P_{\mathrm{AsI}_{3}}^{*}=$ $1,3 \times 10^{-2} \mathrm{~atm} ; P_{\mathrm{GeI}_{2}}^{*}=1,3 \times 10^{-2} \mathrm{~atm}$.

[ $\beta$ as a function of $\log K .1 . P_{\mathrm{AsI}_{3}}^{*}=1.3 \times 10^{-3} \mathrm{~atm}$; $P_{\mathrm{GeI}_{2}}^{*}=1.3 \times 10^{-2} \quad \mathrm{~atm} ; 2 . P_{\mathrm{AsI}_{3}}^{*}=1.3 \times 10^{-3} \quad \mathrm{~atm}$; $P_{\mathrm{Gel}_{2}}^{*}=6.6 \times 10^{-3} \mathrm{~atm} ; 3 . P_{\mathrm{AsI}_{3}}^{*}=1.3 \times 10^{-3} \mathrm{~atm}$; $P_{\text {GeI }_{2}}^{*}=1.3 \times 10^{-2}$ atm. $]$

au niveau de la zone de dépôt. En effet, on peut considérer la réaction d'attaque du GaAs par $I_{2}$ sous la forme :

$$
\begin{aligned}
2 \mathrm{GaAs}+\mathrm{I}_{2}(\mathrm{~g}) & \leftrightarrows 2 \mathrm{GaI}(\mathrm{g})+1 / 2 \mathrm{As}_{4}(\mathrm{~g}) \\
\mathrm{As}_{4}(\mathrm{~g}) & \leftrightarrows 4 \mathrm{As}(\mathrm{s})
\end{aligned}
$$

en ne considérant ici que l'intervention de l'arsenic. Cette attaque chimique du substrat a été vérifiée expérimentalement : d'une part par une étude de l'attaque de GaAs par $\mathrm{I}_{2}+\mathrm{H}_{2}$ en l'absence de source qui a montré que la perte en poids des substrats augmente légèrement lorsque la température de dépôt augmente ou lorsque la pression partielle de l'iode augmente, d'autre part par une étude à la sonde ionique dans la couche qui a montré l'existence d'une incorporation d'arsenic dans la couche et d'une perte de gallium [11].

\section{Etude en fonction de la température.}

Nous avons représenté sur la figure 6 la variation de $R_{\mathrm{H}}, \rho$ et $\mu_{\mathrm{H}}$ en fonction de $10^{3} / T$ pour un échantillon $\mathrm{Ge}(\mathrm{n}) / \mathrm{GaAs}$.

Ces courbes sont typiques de ce que nous obtenons pour ce type d'échantillons après dopage $n$ par $\mathrm{AsH}_{3}$, que le substrat soit du Ge ou du GaAs. 


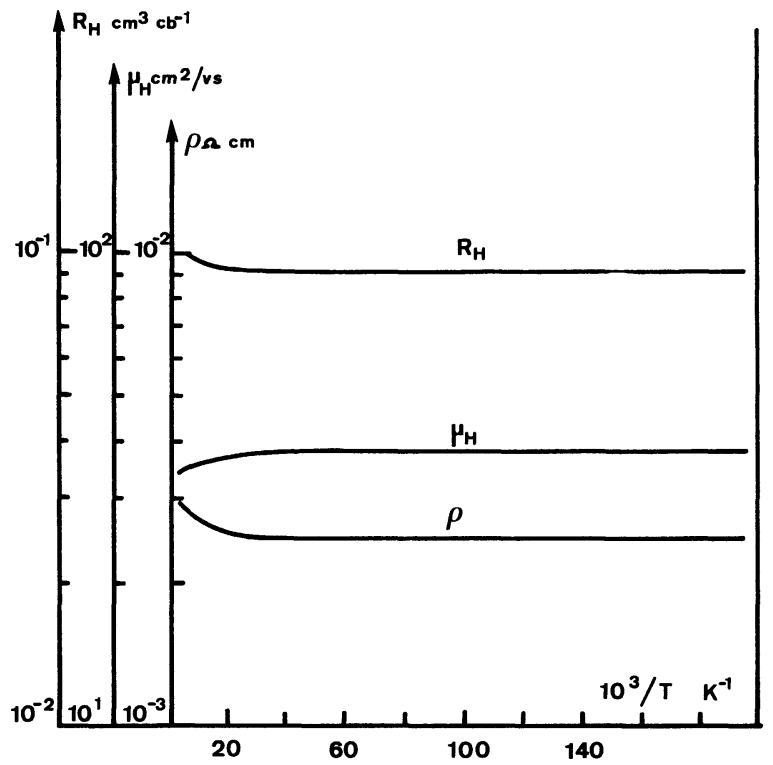

Fig. 6. $-\mathrm{Ge}(\mathrm{n}) / \mathrm{Ge}$. - Variation de $R_{\mathrm{H}}, \rho$ et $\mu_{\mathrm{H}}$ en fonction de $10^{3} / T$.

$\left[\mathrm{Ge}(\mathrm{n}) / \mathrm{Ge}: R_{\mathrm{H}}, \rho\right.$ and $\mu_{\mathrm{H}}$ as a function of $\left.10^{3} / \mathrm{T}.\right]$
Le taux de dopage étant très élevé, nous n'obtenons pas de variations avec la température de $R_{\mathrm{H}}, \rho$ et $\mu_{\mathrm{H}}$, ce qui caractérise une conduction de type métallique.

\section{Conclusion.}

Le taux de dopage $n$ avec les paramètres de fabrication utilisés est assez important même à faible débit de $\mathrm{AsH}_{3}$ rendant ainsi difficile le contrôle rigoureux des paramètres de dopage, du fait de l'incorporation supplémentaire d'arsenic dû à l'attaque chimique du substrat par formation de $\mathrm{AsI}_{3}$ et réactivité en présence de $\mathrm{GeI}_{2}$. Il est à noter qu'un dopage du type $\mathbf{n}$ compris entre $10^{18}$ et $10^{20}$ correspondrait dans le cas d'une jonction $\mathrm{p}-\mathrm{n}$, à la réalisation d'une jonction tunnel non compatible avec l'utilisation de la jonction en photopile. En fait, même avec une dilution de 100 ppm d'arsine dans l'hydrogène, des débits de $\mathrm{AsH}_{3}$ très faibles et des températures aussi basses que $350^{\circ} \mathrm{C}$, le dopage $\mathrm{n}$ obtenu est élevé et à la limite des valeurs acceptables pour la réalisation d'une jonction p-n.

\section{Bibliographie}

[1] Reisman, A., Alyanakyan, S. A., J. Electrochem. Soc. 111 (1964) 1154.

[2] Berkenblit, M., Reisman, A., Light, T. B., J. Electrochem. Soc. 115 (1968) 966.

[3] Riben, A. R., Fencht, D. L., Oldham, W. G., J. Electrochem. Soc. 115 (1966) 245.

[4] Seltzer, M. S., Albon, N., Paris, B., Himes, R. C., J. Electrochem. Soc. 114 (1967) 102.

[5] Kolesnikov, V. N., Danisyena, E. A., DCreza, L. I., Sov. Phys. Crystallog. 12 (1979) 975.

[6] Reisman, A., Berkenblit, M., J. Electrochem. Soc. 112 (1965) 315.
[7] Reisman, A., Berkenblit, M., J. Electrochem. Soc. 113 (1966) 146.

[8] Namordi, M. R., Shaw, D. W., DOeCheCK, F. H., I.E.E.E. Trans Electron. Devices ED 26(1979) 1074.

[9] Etienne, D., Bougnot, G., J. Crystal Growth 69 (1984) 499.

[10] Etienne, D., Bougnot, G., Achargui, N., J. Crystal Growth, à paraître.

[11] Etienne, D., Bougnot, G., Thin Solid. Films, à paraître. 